\title{
CORRECTION OF ORIENTAL EPIBLEPHARON BY ANTERIOR LAMELLAR REPOSITION
}

\author{
CHAITECK CHOO \\ Singapore
}

\begin{abstract}
SUMMARY
Epiblepharon commonly occurs in Oriental infants and children, but usually corrects itself with age. Epiblepharon in adults is not uncommon and may be misdiagnosed or detected late. It causes symptoms of ocular irritation and inferior punctate corneal epithelial erosion. These can persist if surgical treatment is ineffective. A series of 15 lower lid epiblepharon in 10 adults is presented with the clinical features and surgical treatment by anterior lamellar reduction and repositioning. The surgical technique is discussed. All patients had significant symptomatic relief and correction of lash-corneal touch. Among 15 cases of epiblepharon, 3 with residual trichiasis post-operatively were further corrected by skin reduction and debulking of pretarsal orbicularis oculi to achieve total correction. Adequate skin reduction and debulking of orbicularis oculi, especially the pretarsal muscle, are essential in the anterior lamellar repositioning and effective correction of this condition.
\end{abstract}

Epiblepharon is frequently encountered in Oriental eyelids, especially in children. It is characterised by a horizontal fold of redundant skin and abundant orbicularis muscle near the lid margin, which roll the lashes vertically, or posteriorly towards the eye. The tarsal plate and lid margin position remain normal. Epiblepharon may be well tolerated despite the eyelashes being rolled against the cornea. It tends to disappear spontaneously with age and development of the lid. In a Japanese study, 2\% of high school students were found to have epiblepharon. ${ }^{1}$ Surgical therapy of epiblepharon in correcting the trichiasis includes lid bracing sutures, ${ }^{2}$ buried sutures, skin resection, ${ }^{3}$ a Hotz type procedure ${ }^{4}$ or its modification, ${ }^{5,6}$ and a Jones procedure. ${ }^{7}$

Epiblepharon is not uncommon in adults; it may he misdiagnosed or detected late. This paper

Correspondence to: Dr ChaiTeck Choo, FRCOphth, Singapore National Eye Centre, 11, Third Hospital Avenue, Singapore 168751. describes 15 cases of epiblepharon in 10 adult patients, together with the presentation and the surgical treatment. It emphasises the importance of adequate skin reduction and pretarsal orbicularis oculi debulking to achieve proper anterior lamellar repositioning and hence correction of the condition.

\section{SUBJECTS AND METHODS}

The case notes of 10 consecutive Chinese adults (15 lids) who underwent epiblepharon surgery in the period between January 1992 and August 1994 were reviewed. Surgery was performed with the patient under local anaesthesia. The mean age of the group was 22 years (range 15-32 years). There were 8 females and 2 males. The patient details are shown in Table I. All except one case (no. 10) had lashcorneal touch. The exception had skin-corneal touch on downgaze. This patient, who was a foreign worker, had had cryotherapy in her own country for removal of trichiasis. All the cases of lower lid trichiasis were corrected by anterior lamellar reduction and repositioning.

\section{Surgical Techniques}

The amount of lower lid excess skin to be removed is drawn' on the lid after pinching the skin gently with forceps without causing ectropion. The upper skin incisional line is $2-3 \mathrm{~mm}$ from the lid margin, and the delineated ellipse is more on the nasal half of the lower lid. Local anaesthetic agent is then infiltrated subcutaneously. The ellipse of redundant musculocutaneous tissue is excised with blade and scissors (Fig. 1). The anterior lamellar flap is raised after exposing the anterior tarsal surface. Further debulking of the pretarsal orbicularis oculi is achieved by undermining the skin of the anterior lamellar flap until the lash root is just seen. The underlying pretarsal orbicularis muscle is dissected from the superior skin flap.

Care is taken not to damage the hair root or to button-hole the skin. Immediate lash eversion is seen 
Table I. Patient data

\begin{tabular}{|c|c|c|c|c|c|c|}
\hline Patient & $\begin{array}{l}\text { Sex/age } \\
\text { (years) }\end{array}$ & Symptoms & Signs & Operation & $\begin{array}{c}\text { Follow-up } \\
\text { (months) }\end{array}$ & Result \\
\hline 1 & $\mathrm{~F} / 27$ & $\mathrm{R}$ tearing/photophobia & spk & R/L ALR & 36 & $\mathrm{R} / \mathrm{L}$ good \\
\hline 2 & $\mathrm{~F} / 26$ & $\mathrm{R}$ ocular irritation & spk & $\mathrm{R} / \mathrm{L}$ ALR & 7 & $\begin{array}{l}\mathrm{L} \text { good } \\
\mathrm{R} \text { recurrence }\end{array}$ \\
\hline 3 & $\mathrm{~F} / 17$ & $\mathrm{R} / \mathrm{L}$ tearing & spk & R/L ALR & 6 & $\mathrm{R} / \mathrm{L}$ good \\
\hline 4 & $\mathrm{~F} / 18$ & L ocular irritation & spk & L/ALR & 7 & $\mathrm{~L}$ good \\
\hline 5 & M/19 & L CL fitting problem & spk & L/ALR & 11 & $\mathrm{~L}$ good \\
\hline 6 & $\mathrm{~F} / 32$ & L ocular irritation & spk & L/ALR & 14 & $\mathrm{~L}$ good \\
\hline 7 & $\mathrm{~F} / 19$ & RL CL fitting problem & spk & R/L ALR & 7 & $\mathrm{RL}$ recurrence \\
\hline 8 & $\mathrm{M} / 15$ & RL tearing /discomfort & spk & $\mathrm{R} / \mathrm{L}$ ALR & 6 & $\mathrm{R} / \mathrm{L} \operatorname{good}$ \\
\hline 9 & $\mathrm{~F} / 26$ & $\mathrm{R}$ discomfort & spk & R ALR & 6 & $\mathrm{R}$ good \\
\hline 10 & $\mathrm{~F} / 21$ & $\mathrm{R}$ ocular irritation & spk & R ALR & 6 & $\mathrm{R}$ good \\
\hline
\end{tabular}

$\mathrm{R}$, right; L, left; spk, superficial punctate keratitis; ALR, anterior lamellar reposition; CL, contact lens.

when the anterior lamella is repositioned on the tarsal surface before suturing the tissues. Subcutaneous $7 / 0$ absorbable sutures are used to hold the anterior lamella onto the anterior tarsus, but no attempt is made to suture inferior retractor (Fig. 2). The two skin edges are gently apposed with $7 / 0$ silk without skin crease formation, as the lower lids of most Chinese patients do not have a skin crease. Post-operatively antibiotic ointment is applied to the skin wound. Skin sutures are removed on the fifth postoperative day.

\section{RESULTS}

Fifteen lower lids of 10 patients were operated on. Patients were followed up for a minimum of 6 months (range 6-34 months). All the cases were reviewed on the first post-operative day, fifth postoperative day, first post-operative month and sixth post-operative month. Seven patients complaining pre-operatively of ocular irritation and/or tearing had significant symptomatic relief. Two patients who had contact lens fitting problems were able to use contact lenses again. All patients had correction of lashcorneal touch and their cosmetic results were good.

Corneal superficial punctate epithelial erosions resolved in all cases examined on the fifth postoperative day by slit lamp microscopy. Three lids

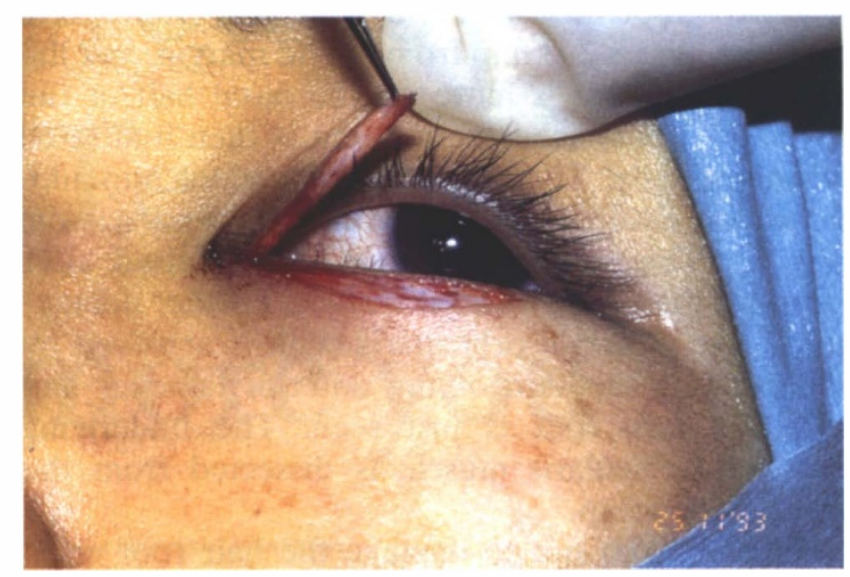

Fig. 1. Excision of redundant skin muscle flap with blade and scissors. (two patients: nos. 2 and 7) of 15 cases of epiblepharon that were found to have residual trichiasis within 6 months were corrected by further skin reduction and debulking of the pretarsal orbicularis oculi to achieve total correction. After the second procedure, these two patients were again reviewed on the first and fifth post-operative day, and the first and sixth post-operative month. The residual trichiasis was completely corrected with good cosmesis and no recurrences were observed.

\section{DISCUSSION}

Epiblepharon is due to an excessive fold of skin with abundant underlying orbicularis muscle which pushes the lower lid lashes vertically or towards to the cornea. The tarsal plate position is normal in epiblepharon. If inturning of the lashes in epiblepharon is due to excess skin and its underlying abundant or hypertrophied pretarsal orbicularis oculi, then the logical surgery is skin reduction and muscle debulking to evert the lashes without creating a skin crease and ectropion. Johnson and Semple 8 described excision of much of the hypertrophied muscle strip as a more effective way of correcting epiblepharon in Eskimos after analysing seven cases in their report. They stressed the importance of making a distinction between epiblepharon and congenital entropion, as the operative treatments of

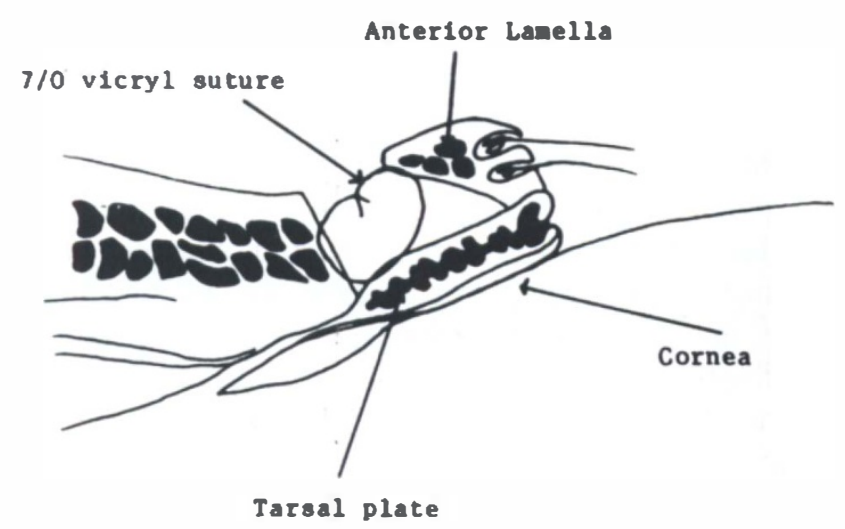

Fig. 2. Schematic drawing of anterior lamellar repositioning after pretarsal orbicularis debulking and placement of subcutaneous sutures. 


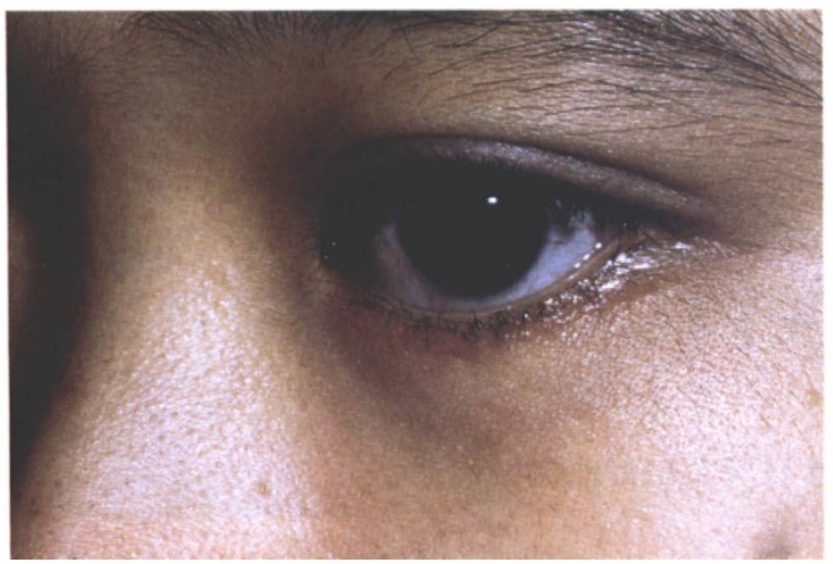

Fig. 3. Patient 4. Lash eversion, no skin crease and good cosmesis after surgery.

the two are different. Since epiblepharon is a relatively common problem among Orientals and a lower lid crease is not commonly present, the approach to this condition may be looked at differently. There has been no report emphasising pretarsal orbicularis debulking in the repair of epiblepharon in Chinese patients. I have found that this is the important step in correcting this anomaly after appropriate skin reduction.

All 15 lids treated by anterior lamellar reposition achieved correction of lash-corneal touch and good cosmesis (Fig. 3). No attempt was made to create a lid crease as this was not an essential feature in this series of patients, or to identify and plicate the inferior retractor, or to reinforce the inferior retractor (CCPF) attachment, as it is believed to be normal in Chinese epiblepharon patients. Among 15 lids, 3 with residual trichiasis post-operatively were further improved by repeating the same procedures, as the patients were found still to have redundant skin and pretarsal orbicularis muscle. Intraoperatively this confirmed that the first surgery had been too conservative in removing the orbicularis under the superior skin flap. After the repeat procedure, the residual trichiasis was completely corrected. No recurrences were detected when the patients were reviewed at the sixth post-operative month.

The relationship of septum and inferior retractor in the lower lid is analogous to the relationship of septum and levator aponeurosis in the upper lid. Orientals have an exaggerated upper lid skin fold and a poor upper lid crease due to more inferior insertion of the orbital septum onto the levator aponeurosis. ${ }^{9}$ Absence of a poor or lower lid crease in a Chinese patient is presumably due to a deficiency in cutaneous-capsulopalpebral fibre attachment resulting from the higher septum attachment onto the tarsus or inferior retractor. Quickert et $a l^{2}$ hypothesised that epiblepharon is caused by congenital absence of retractor fibres into the skin and orbicularis or by higher retractor fibre insertion nearer the eyelid margin. The anterior lamella is therefore not firmly held against the tarsal plate. Together with the exaggerated skin fold and abundant pretarsal muscle, the anterior lamella is easily rolled upwards and away from the tarsal plate. Naturally the cilia originating posterior to the marginal pretarsal orbicularis are tilted and directed towards the globe. Therefore adequate skin reduction and debulking of orbicularis oculi, especially the pretarsal muscle, are essential in anterior lamellar repositioning and effective correction of this condition. The same procedure has since been applied in correcting epiblepharon in children under general anaesthesia with good results.

In conclusion, the principles of this surgical procedure for the correction of trichiasis in Oriental epiblepharon are adequate excision of a strip of pretarsal orbicularis muscle and placement of buried sutures to reposition the anterior lamella. Hence not only is the lower lid skin wound closed without formation of a lid crease, but an effective lash eversion with good cosmesis is also achieved in the Oriental patient.

Key words: Anterior lamellar reposition, Epiblepharon, Pretarsal orbicularis, Trichiasis.

\section{REFERENCES}

1. Noda S, Hayasaka S, Setogawa T. Epiblepharon with inverted eyelashes in Japanese children. I. Incidence and symptoms. Br J Ophthalmol 1989;73:126-7.

2. Quickert MH, Wilkes DI, Dryden RM. Nonincisional correction of epiblepharon and congenital entropion. Arch Ophthalmol 1983;101:778-81.

3. Hayasaka S, Noda S, Setogawa T. Epiblepharon with inverted eyelashes in Japanese children. II. Surgical repairs. Br J Ophthalmol 1989;73:128-30.

4. Hotz FC. A new operation for entropion and trichiasis. Arch Ophthalmol 1879;8:249-63.

5. O'Donnell BA, Collin JRO. Congenital lower eyelid deformity with trichiasis (epiblepharon and entropion). Aust NZ J Ophthalmol 1994;22:33-7.

6. Collin JRO: A manual of systematic eyelid surgery, 2nd ed. Ednburgh: Churchill Livingstone, 1989:chap. 2 .

7. Millman AL, Mannor GE, Putterman AM. Lid crease and capsulopalpebral fascia repair in congenital entropion and epiblepharon. Ophthalmic Surg 1994;25:1625.

8. Johnson GJ, Semple J. Epiblepharon in Labrador. Can J Ophthalmol 1977;12:175-81.

9. Doxanas MT, Anderson RL. Clinical orbital anatomy. Baltimore: Williams and Wilkins, 1984:chap.4. 